\title{
Fatores associados à Hipertensão Arterial Sistêmica autorreferida segundo VIGITEL nas 26 capitais brasileiras e no Distrito Federal em 2008
}

\author{
Factors associated with self-reported systemic arterial \\ hypertension according to VIGITEL in 26 Brazilian capitals \\ and the Federal District in 2008
}

Ana Paula Muraro ${ }^{1}$

Debora França dos Santos ${ }^{1}$

Paulo Rogério Melo Rodrigues ${ }^{2}$

José Ueleres Braga ${ }^{1}$

${ }^{1}$ Departamento de Epidemiologia, Instituto de Medicina Social, Universidade do Estado do Rio de Janeiro. R. São Francisco Xavier 524/7\%/Bloco E, Maracanã. 20550-012 Rio de Janeiro RJ. muraroap@gmail.com ${ }^{2}$ Departamento de Nutrição Social e Aplicada, Instituto de Nutrição Josué de Castro, Universidade Federal do Rio de Janeiro.

\begin{abstract}
The scope of this article is to study the association between lifestyle, nutritional status and the prevalence of self-reported systemic arterial hypertension, weighted by the system of risk and protective factors for Chronic Non-Communicable Diseases (CNCD) by telephone surveys and standardized by age and sex, in adults from 26 Brazilian state capitals and the Federal District in 2008. For each city the prevalence of hypertension was standardized by the direct method. Multiple linear regression analysis was performed between the factors of interest and the prevalence of hypertension, weighted and standardized by gender and age. After standardization, the prevalence of hypertension tended to increase in capitals with a younger population and decrease in those with a higher proportion of elderly individuals. In regression models, the prevalence of weighted and standardized hypertension remained associated with the prevalence of excess weight and the consumption of fruit and vegetables $(F V)$. However, physical activity was only negatively associated with the weighted prevalence of hypertension. A positive association with weighted and standardized prevalence of hypertension was observed with excess weight and regular consumption of $F V$.
\end{abstract}

Key words Hypertension, Risk factors, Ecological studies, Telephone surveys
Resumo O objetivo deste artigo é estudar a associação entre estilo de vida e estado nutricional e prevalência de Hipertensão Arterial Sistêmica (HAS) autorreferida, ponderada pelo sistema de Vigilância de Fatores de Risco e Proteção para Doenças Crônicas por Inquérito Telefônico (VIGITEL) e padronizada por idade e sexo, em indivíduos adultos das 26 capitais brasileiras e Distrito Federal em 2008. Calculou-se para cada capital a prevalência de HAS padronizada pelo método direto. Realizou-se regressão linear múltipla dos fatores de interesse em relação à prevalência de HAS ponderada e padronizada por sexo e idade. Após a padronização, a prevalência de HAS tendeu a aumentar nas capitais com população mais jovem e a diminuir naquelas com mais idosos. Nos modelos de regressão, mantiveram-se associados à prevalência de HAS, ponderada e padronizada, o excesso de peso e o consumo de frutas e hortaliças. Porém, a atividade física associou-se negativamente apenas à prevalência de HAS ponderada. Observou-se, com a prevalência ponderada e padronizada de HAS, associação positiva com excesso de peso e consumo regular de frutas e hortaliças e apenas com a prevalência ponderada observou-se associação inversa dessa morbidade com a prática de atividade física.

Palavras-chave Hipertensão, Fatores de risco, Estudos ecológicos, Inquéritos telefônicos 


\section{Introdução}

A hipertensão arterial sistêmica (HAS) é considerada como o principal fator de risco para doenças cardiovasculares, sendo também um dos principais problemas de saúde pública, levando a um elevado custo médico-social ${ }^{1,2}$.

No Brasil, a prevalência de HAS estimada em 2008 foi de $24,0 \%$ entre as mulheres e $17,3 \%$ entre os homens com idade $\geq 20$ anos, cerca de metade dos homens e mais da metade das mulheres com idade $\geq 60$ anos relataram diagnóstico prévio de hipertensão ${ }^{3}$. Verifica-se ainda que a prevalência de HAS autorrelatada aumentou uniformemente de 2006 a 2009 entre os brasileiros, passando de 21,5 para $24,4 \%{ }^{4}$. O monitoramento dessa morbidade, portanto, torna-se importante para o controle das doenças cardiovasculares que, apesar de terem diminuído, ainda são a principal causa de morte o Brasil ${ }^{5}$.

Vários são os fatores que podem estar associados ao aumento da prevalência de HAS, tais como sedentarismo, estresse, tabagismo, envelhecimento, gênero, aumento do peso corporal e fatores dietéticos ${ }^{6}$. Entre estes, um aspecto que merece destaque na população brasileira é a modificação dos hábitos alimentares e de estilo de vida, como a redução prática de atividade física, que indica exposição cada vez mais intensa a riscos cardiovasculares ${ }^{7,8}$.

A vigilância de fatores de risco para as Doenças Crônicas Não Transmissíveis (DCNT) permite detectar tendências no tempo e no espaço geográfico, possibilitando o planejamento de ações preventivas em saúde pública. Apesar de estar sujeito a erros de classificação, o autorrelato do diagnóstico de HAS é uma estratégia de baixo custo, acessível e de rápida aplicação, já validado para utilização em estudos populacionais como indicador apropriado para estimar a prevalência de $\mathrm{HAS}^{9,10}$.

Como parte do processo de dotar o país de instrumentos eficazes para monitorar a frequência e a distribuição dos principais fatores determinantes das DCNT, o Ministério da Saúde implantou, no ano de 2006, o sistema VIGITEL - Vigilância de Fatores de Risco e Proteção para Doenças Crônicas por Inquérito Telefônico ${ }^{11}$. Este objetiva o monitoramento contínuo da frequência e distribuição de fatores de risco e de proteção para doenças crônicas nas 26 capitais brasileiras e no Distrito Federal, apoiando-se na realização contínua de entrevistas telefônicas em amostras probabilísticas da população adulta (indivíduos maiores de 18 anos de idade) servidos por linhas fixas de telefone ${ }^{12}$.
Este trabalho teve como objetivo estudar as relações entre as características do estilo de vida e estado nutricional e as prevalências de HAS autorreferida ponderada pelo VIGITEL e padronizada por idade e sexo, nas 26 capitais brasileiras e no Distrito Federal. Contribuindo assim para o entendimento dessas relações em nível populacional e para elaboração de estratégias regionais e nacionais direcionadas à população adulta.

\section{Métodos}

Trata-se de um estudo ecológico, tendo como unidade de análise as capitais dos 26 estados brasileiros e o Distrito Federal e como desfechos, a prevalência de HAS autorreferida ponderada pelo VIGITEL e a padronizada por sexo e idade pela população total das 26 capitais brasileiras e Distrito Federal, no ano de 2008.

Neste estudo, foram usados dados secundários de acesso universal e disponíveis no site DATASUS ${ }^{13}$.

\section{População estudada e local do estudo}

Foi avaliada a população adulta das 26 capitais brasileiras e do Distrito Federal, no ano de 2008, obtida pelo VIGITEL.

Os procedimentos de amostragem empregados pelo VIGITEL visam obter amostras probabilísticas da população de adultos residentes em domicílios servidos por pelo menos uma linha telefônica fixa no ano. O mínimo de 2.000 indivíduos com 18 anos ou mais de idade em cada cidade é estabelecido como o tamanho amostral, para que se possa estimar com coeficiente de confiança de $95 \%$ e erro máximo de cerca de dois pontos percentuais a frequência de qualquer fator de risco na população adulta ${ }^{12}$. No ano de 2008, as ligações feitas para as 72.834 linhas elegíveis corresponderam a 54.353 entrevistas completas, o que indica taxa média de sucesso do sistema de $74,6 \%{ }^{12}$.

As medidas obtidas pelo VIGITEL são ponderadas para ajustar a distribuição sociodemográfica da amostra à distribuição da população adulta de cada cidade no Censo Demográfico de 2000 e para considerar seu peso populacional, permitindo a correção da sub ou super-representação de estratos sociodemográficos, decorrente da cobertura diferencial desses estratos pela rede telefônica. O processo de amostragem e detalhamento dos métodos empregados no VIGITEL pode ser obtido nas publicações do Ministério da Saúde ${ }^{12}$. 


\section{Acesso aos dados}

Os dados sobre a prevalência de HAS e os fatores de risco e proteção avaliados neste estudo foram obtidos por meio do sistema VIGITEL no ano de 2008, acessados a partir do site do DATASUS $^{13}$. Para o processamento das informações, incluindo obtenção, montagem e organização dos dados, foi utilizado o programa TABWIN do Ministério da Saúde.

Para realizar a padronização da prevalência de HAS pelo método direto, adotou-se como padrão a população total das 27 cidades avaliadas por meio dos dados oriundos das estimativas do IBGE por cidade, estratificada por faixa etária e sexo, com data de referência de $1^{\circ}$ de julho de 2008 e elaboradas no âmbito do Projeto UNFPA/IGBE - População e Desenvolvimento Coordenação de População e Indicadores Sociais. Cabe ressaltar que a margem de erro foi maior para municípios de pequeno contingente populacional, principalmente ao estratificar os dados. Essas informações também são de acesso universal disponíveis no site do DATASUS ${ }^{13}$.

Apesar de o VIGITEL utilizar para a ponderação de suas estimativas o censo populacional do IBGE de 2000, utilizou-se neste estudo a estimativa de 2008 para a padronização por assumir que não houve mudanças significativas na população quanto à distribuição etária e de gênero. Como exemplo, de acordo com os dados do IBGE disponíveis no site do DATASUS ${ }^{13}$, no censo de 2000 , cerca de $53,9 \%$ da população das 26 capitais brasileiras e distrito federal eram mulheres e $13,1 \%$ na faixa etária entre 25 e 29 anos, sendo estimado em 2008 a proporção de 54,0\% de mulheres e 13,7\% nesta mesma faixa etária.

\section{Variáveis estudadas}

As variáveis desfechos do presente estudo foram a prevalência de HAS autorreferida ponderada pelo VIGITEL e a padronizada por sexo e idade pela população total das 26 capitais brasileiras e Distrito Federal, no ano de 2008. Como variáveis características de estilo de vida: tabagismo (proporção de indivíduos que fumam mais de vinte cigarros por dia); consumo recomendado de frutas e hortaliças (proporção de indivíduos que consomem pelo menos 5 porções de frutas e hortaliças por dia em 5 ou mais dias na semana); consumo de excesso de gordura da carne (proporção de indivíduos que consomem carne gordurosa ou frango com pele, sem remover a gordura visível); consumo frequente de refrigerante não diet (proporção de indivíduos que cos- tumam consumir refrigerante ou suco artificial com açúcar cinco ou mais dias por semana); pratica regular de atividade física (proporção de indivíduos que praticam atividades físicas de intensidade leve ou moderada por pelo menos trinta minutos diários em cinco ou mais dias por semana ou atividades de intensidade vigorosa por pelo menos vinte minutos diários em três ou mais dias da semana) e consumo abusivo de álcool (proporção de indivíduos que, nos últimos 30 dias, consumiram mais do que quatro [mulher] ou cinco [homem] doses de bebida alcoólica em uma mesma ocasião). Variáveis indicadoras do estado nutricional foram: excesso de peso (proporção de indivíduos com índice de massa corporal IMC - igual ou superior a $25 \mathrm{~kg} / \mathrm{m}^{2}$ ) e obesidade (proporção de indivíduos com índice de massa corporal igual ou superior a $30 \mathrm{~kg} / \mathrm{m}^{2}$ ). Foi avaliada também a proporção de indivíduos que possuíam plano de saúde ou convênio médico.

\section{Análise dos dados}

Foram estimadas as prevalências pontuais e seus intervalos de confiança de 95\% de HAS autorreferida segundo sexo e faixa-etária, além das proporções das características demográficas e estilo de vida das 26 capitais brasileiras e do Distrito Federal.

Entre as características populacionais das 26 capitais brasileiras e Distrito Federal, notou-se diferença quanto à distribuição da sua estrutura etária, por esse motivo, para cada capital foi calculada a prevalência de HAS padronizada pelo método direto, adotando como padrão a distribuição por sexo e idade da população total das 26 capitais brasileiras e do Distrito Federal.

Após a padronização, realizou-se regressão linear univariada, para verificar a existência de associações entre as dependentes, HAS autorreferida ponderada e padronizada, e os fatores socioeconômicos, de estilo de vida e de estado nutricional estudados. Nesta etapa, foram identificadas as variáveis que apresentaram associação ao nível de significância de $20 \%$, sendo incluídas e mantidas nos modelos de regressão múltipla.

Procedeu-se análise de correlação de Pearson para avaliar a ocorrência de colinearidade entre as variáveis explicativas. $\mathrm{Na}$ regressão linear múltipla foram considerados significativos os valores de $\mathrm{p}$ menores que 0,05 . Por fim, foram calculados os coeficientes de determinação (R2) dos modelos múltiplos.

Para as análises estatísticas o pacote estatístico "R" versão 2.12.2., projeto colaborativo disponível gratuitamente na Internet ${ }^{14}$. 


\section{Considerações éticas}

O presente estudo foi aprovado pelo Comitê de Ética em Pesquisa do Instituto de Medicina Social da Universidade do Estado do Rio de Janeiro em 9 de novembro de 2011.

\section{Resultados}

A proporção de indivíduos adultos com mais de 18 anos de idade que referiram diagnóstico médico de HAS foi de $23,1 \%$, sendo ligeiramente maior entre as mulheres $(25,5 \%)$ do que entre os homens (20,3\%). Essa prevalência variou entre $14,7 \%$ em Palmas e 29,5\% no Rio de Janeiro.

O diagnóstico de HAS se torna mais comum com o aumento da idade, alcançando as maiores prevalências entre os 18 e os 24 anos de idade em Teresina (10,6), Porto Alegre (10,6\%) e Manaus (10,1\%). Na faixa etária de 65 anos ou mais as maiores prevalências de HAS foram verificadas em Goiânia (66,2\%), Aracajú (65,0\%) e Curitiba (64,9\%) (Tabela 1).

Após a padronização da prevalência de HAS autorreferida, realizada pelo método direto por sexo e faixa etária da soma das 26 capitais brasileiras e Distrito Federal, nota-se que nas cidades cuja população era predominantemente jovem ou adulto jovem essa prevalência tendeu a aumentar. No entanto, em Porto Alegre e Rio de Janeiro, cidades caracterizadas por maior proporção de idosos, essa prevalência diminuiu após a padronização (Tabela 2 ).

A prevalência de adultos que referiram praticar atividade física no tempo livre foi relativamen-

Tabela 1. Prevalência (\%) de adultos ( $\geq 18$ anos) que referiram diagnóstico médico de Hipertensão Arterial Sistêmica ${ }^{a}$, segundo as 26 capitais brasileiras e Distrito Federal, por faixa etária, VIGITEL, 2008.

\begin{tabular}{|c|c|c|c|c|c|c|c|}
\hline \multirow[b]{2}{*}{ Capitais } & \multicolumn{7}{|c|}{ Prevalência VIGITEL } \\
\hline & Total & $\begin{array}{c}18 \text { a } 24 \\
\text { anos }\end{array}$ & $\begin{array}{c}25 \text { a } 34 \\
\text { anos }\end{array}$ & $\begin{array}{c}35 \text { a } 44 \\
\text { anos }\end{array}$ & $\begin{array}{c}45 \text { a } 54 \\
\text { anos }\end{array}$ & $\begin{array}{c}55 \text { a } 64 \\
\text { anos }\end{array}$ & $\begin{array}{c}65 \text { anos } \\
\text { e mais }\end{array}$ \\
\hline Aracaju & 21,9 & 5,7 & 7,0 & 18,9 & 39,2 & 59,3 & 65,0 \\
\hline Belém & 17,3 & 2,7 & 10,0 & 15,6 & 30,0 & 42,0 & 49,2 \\
\hline Belo Horizonte & 24,5 & 3,6 & 11,2 & 20,5 & 40,8 & 54,9 & 60,8 \\
\hline Boa Vista & 18,9 & 6,9 & 20,1 & 14,4 & 26,7 & 42,2 & 57,3 \\
\hline Campo Grande & 24,2 & 7,7 & 14,0 & 18,1 & 39,5 & 58,1 & 57,3 \\
\hline Cuiabá & 22,3 & 6,3 & 11,1 & 23,3 & 35,6 & 56,9 & 61,0 \\
\hline Curitiba & 21,2 & 2,7 & 10,9 & 18,9 & 30,1 & 43,6 & 64,9 \\
\hline Florianópolis & 21,7 & 8,6 & 8,8 & 18,3 & 30,2 & 44,4 & 63,3 \\
\hline Fortaleza & 20,0 & 3,6 & 9,6 & 17,0 & 34,5 & 49,9 & 54,7 \\
\hline Goiânia & 18,9 & 1,9 & 7,7 & 16,5 & 31,5 & 50,0 & 66,2 \\
\hline João Pessoa & 24,1 & 6,3 & 7,0 & 25,6 & 41,1 & 50,9 & 64,8 \\
\hline Macapá & 17,6 & 7,3 & 6,6 & 18,6 & 37,0 & 49,6 & 59,7 \\
\hline Maceió & 21,8 & 10,0 & 13,1 & 17,3 & 33,8 & 49,3 & 56,5 \\
\hline Manaus & 16,3 & 10,1 & 8,0 & 14,3 & 28,5 & 35,5 & 54,0 \\
\hline Natal & 24,5 & 2,6 & 15,8 & 25,3 & 38,8 & 48,0 & 59,7 \\
\hline Palmas & 14,7 & 7,2 & 10,9 & 12,0 & 25,9 & 47,1 & 64,2 \\
\hline Porto Alegre & 26,2 & 10,6 & 12,8 & 19,4 & 33,4 & 45,6 & 59,2 \\
\hline Porto Velho & 19,7 & 7,9 & 14,3 & 14,9 & 38,9 & 45,5 & 54,8 \\
\hline Recife & 26,6 & 8,9 & 13,8 & 26,1 & 34,4 & 51,4 & 63,7 \\
\hline Rio Branco & 22,4 & 7,5 & 14,1 & 23,4 & 37,7 & 49,0 & 62,5 \\
\hline Rio de Janeiro & 29,5 & 7,5 & 14,6 & 23,6 & 37,9 & 54,3 & 62,6 \\
\hline Salvador & 23,7 & 10,0 & 14,8 & 23,0 & 32,6 & 50,6 & 62,3 \\
\hline São Luís & 16,7 & 2,4 & 13,1 & 13,4 & 32,4 & 41,7 & 48,2 \\
\hline São Paulo & 26,3 & 6,7 & 9,1 & 23,7 & 42,4 & 58,0 & 63,0 \\
\hline Teresina & 19,9 & 10,6 & 8,7 & 16,6 & 33,8 & 42,3 & 57,4 \\
\hline Vitória & 23,3 & 6,0 & 14,0 & 17,5 & 34,3 & 48,6 & 58,1 \\
\hline Distrito Federal & 20,4 & 5,4 & 10,6 & 22,6 & 35,5 & 47,9 & 61,7 \\
\hline
\end{tabular}

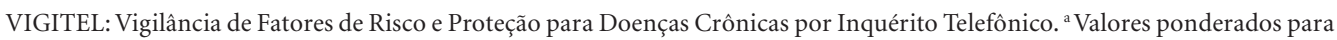
ajustar a distribuição sociodemográfica da amostra VIGITEL à distribuição da população adulta de cada cidade no Censo Demográfico de 2000 . 
te baixa em todas as cidades estudadas, variando entre 11,5\% em São Paulo e 20,6\% em Palmas. O percentual de adultos que referiram consumo abusivo de bebidas alcoólicas nos últimos 30 dias variou entre 10,8\% em Curitiba e 24,9\% em Salvador. A frequência de excesso de peso autorreferido variou entre 36,8\% em Teresina e 49,0\% em Porto Alegre. A proporção de adultos que referiram consumir cinco ou mais porções diárias de frutas e hortaliças variou entre 11,1\% em Belém e 24,1\% em Florianópolis (Tabela 3).

Ao avaliarmos as relações entre cada uma das características de estilo de vida e hábitos alimentares, por meio do coeficiente de correlação de Pearson, observou-se correlação estatisticamente significativa entre tabagismo e: excesso de peso $(\mathrm{r}=0,47 ; \mathrm{p}<0,016)$, consumo de bebida alcoólica $(r=-0,41 ; p<0,033)$, consumo recomendado de frutas e hortaliças $(\mathrm{r}=0,57 ; \mathrm{p}<0,002)$ e plano de saúde $(r=0,55 ; \mathrm{p}<0,003)$; excesso de peso e: consumo de bebida alcoólica $(r=-0,41$; $p$ $<0,035)$ e obesidade $(\mathrm{r}=0,81 ; \mathrm{p}<0,001)$; obesidade e: consumo de bebida alcoólica $(r=-0,41 ; p$ $<0,029$ ); consumo recomendado de frutas e hortaliças e: consumo de bebida alcoólica $(r=-0,39$; $\mathrm{p}<0,041)$ e plano de saúde $(\mathrm{r}=0,73 ; \mathrm{p}<0,001)$ (dados não apresentados). Deste modo, não foram incluídos nos modelos de regressão múltipla os fatores que apresentaram coeficientes de correlação moderada a alta ( $\mathrm{r}$ maior de 0,50 ), sendo eles: proporção de fumantes de mais de 20 cigarros por dia, proporção de obesos e proporção de indivíduos com plano de saúde. Para as variáveis que possuíam elevada correlação, foram mantidas as que apresentaram melhor coeficiente de regressão linear da análise univariada.

$\mathrm{Na}$ Tabela 4 estão apresentadas as variações na prevalência de HAS, obtida por meio da análise de regressão linear, com seus respectivos intervalos de confiança de $95 \%$ e valores de p da relação entre a prevalência de HAS autorreferida e as características do estilo de vida e estado nutricional dos adultos residentes nas 26 capitais brasileiras e Distrito Federal. Nesta tabela observa-se a diferença ao se utilizar prevalências ponderadas e padronizadas para estimar associação entre o desfecho e as variáveis explanatórias, pois, como verificado, a padronização tendeu a atenuar a força de associação entre a prevalência de HAS autorreferida e as variáveis selecionadas.

Após análise da regressão linear e da correlação, foi avaliada a relação entre a HAS autorreferida ponderada e fatores associados, mutuamente ajustados (Tabela 5). Nesta tabela, pode-se observar que a cada unidade da proporção de ex- cesso de peso, ou seja, a cada unidade de proporção de indivíduos acima do IMC de $25 \mathrm{~kg} / \mathrm{m}^{2}$, resulta em aumento de 0,46 na prevalência de HAS autorreferida entre adultos das 26 capitais brasileiras e do Distrito Federal. O mesmo pode ser observado para o consumo recomendado de frutas e hortaliças, para o qual o aumento de cada unidade na proporção deste consumo reflete em aumento de 0,60 na prevalência desta morbidade. Por outro lado, com relação à proporção prática de atividade física suficiente no lazer, pode-se verificar que o aumento desta resulta em diminuição de 0,55 na prevalência de HAS na população avaliada.

Tabela 2. Prevalência (\%) de Hipertensão Arterial Sistêmica auto-referida nas 26 capitais brasileiras e Distrito Federal estimada pelo VIGITEL ${ }^{a}$ e padronizada ${ }^{\text {b }}$, segundo as capitais dos estados brasileiros e Distrito Federal, VIGITEL, 2008.

\begin{tabular}{|c|c|c|c|c|}
\hline \multirow{2}{*}{ Capitais } & \multicolumn{3}{|c|}{ Prevalência do VIGITEL ${ }^{a}$} & \multirow{2}{*}{$\begin{array}{c}\begin{array}{c}\text { Prevalência } \\
\text { padronizada }\end{array} \\
\text { Total }\end{array}$} \\
\hline & Total & Masculino & Feminino & \\
\hline Aracaju & 21,90 & 16,8 & 26,1 & 25,10 \\
\hline Belém & 17,30 & 16,3 & 18,1 & 20,10 \\
\hline Belo Horizonte & 24,50 & 20,2 & 28,2 & 25,60 \\
\hline Boa Vista & 18,90 & 18,7 & 19,1 & 23,70 \\
\hline Campo Grande & 24,20 & 23,7 & 24,6 & 26,60 \\
\hline Cuiabá & 22,30 & 19,3 & 25,0 & 26,30 \\
\hline Curitiba & 21,20 & 17,5 & 24,5 & 22,70 \\
\hline Florianópolis & 21,70 & 19,5 & 23,7 & 23,00 \\
\hline Fortaleza & 20,00 & 18,0 & 21,7 & 22,40 \\
\hline Goiânia & 18,90 & 18,5 & 19,4 & 22,20 \\
\hline João Pessoa & 24,10 & 22,2 & 25,7 & 26,10 \\
\hline Macapá & 17,60 & 13,3 & 21,8 & 23,80 \\
\hline Maceió & 21,80 & 19,4 & 23,8 & 24,50 \\
\hline Manaus & 16,30 & 12,9 & 19,4 & 20,20 \\
\hline Natal & 24,50 & 20,5 & 27,8 & 26,40 \\
\hline Palmas & 14,70 & 15,3 & 14,1 & 21,80 \\
\hline Porto Alegre & 26,20 & 22,3 & 29,4 & 24,70 \\
\hline Porto Velho & 19,70 & 17,4 & 22,0 & 24,50 \\
\hline Recife & 26,60 & 21,6 & 30,5 & 27,20 \\
\hline Rio Branco & 22,40 & 18,1 & 26,4 & 27,00 \\
\hline Rio de Janeiro & 29,50 & 23,4 & 34,6 & 27,50 \\
\hline Salvador & 23,70 & 21,2 & 25,7 & 26,60 \\
\hline São Luís & 16,70 & 12,8 & 20,0 & 20,70 \\
\hline São Paulo & 26,30 & 25,0 & 27,4 & 27,10 \\
\hline Teresina & 19,90 & 16,7 & 22,5 & 22,70 \\
\hline Vitória & 23,30 & 22,9 & 23,7 & 24,30 \\
\hline Distrito Federal & 20,40 & 19,3 & 21,4 & 24,90 \\
\hline
\end{tabular}

VIGITEL: Vigilância de Fatores de Risco e Proteção para Doenças Crônicas por Inquérito Telefônico. ${ }^{\mathrm{a}}$ Valores ponderados para ajustar a distribuição sociodemográfica da amostra VIGITEL à distribuição da população adulta de cada cidade no Censo Demográfico de 2000.

${ }^{\text {b }}$ Padronizada pelo método direto por sexo e faixa etária da soma das 26 capitais brasileiras e Distrito Federal. 
Ainda na Tabela 5, ao avaliar a relação entre a prevalência de HAS autorreferida padronizada e os fatores associados, mutuamente ajustados, pode-se verificar que a associação entre a prevalência dessa morbidade e a prática de atividade física perdeu a significância estatística quando comparada à ponderada. Com relação à associação entre as proporções de excesso de peso e consumo recomendado de frutas e hortaliças com a prevalência de HAS autorreferida houve a atenuação da força de associação (coeficiente da regressão linear), porém mantiveram-se significantes.

\section{Discussão}

No presente estudo verificaram-se associações positivas entre a proporção de excesso de peso e de consumo recomendado de frutas e hortaliças, tanto na análise com a prevalência de HAS ponderada pelo VIGITEL quanto com a padronizada para sexo e idade. No entanto, a associação inversa com a proporção de prática de atividade física suficiente no lazer não foi mantida após a padronização, indicando que a associação verificada inicialmente, com a prevalência de HAS pon-

Tabela 3. Proporção (\%) de adultos ( $\geq 18$ anos) que praticam atividade física no tempo livrea , consumo abusivo de álcool ${ }^{\mathrm{b}}$, com IMC acima de $25 \mathrm{~kg} / \mathrm{m}^{2 \mathrm{c}}$ e consumo de frutas e hortaliças ${ }^{\mathrm{d}}$ segundo as 26 capitais brasileiras e Distrito Federale, VIGITEL, 2008.

\begin{tabular}{|c|c|c|c|c|c|c|c|c|}
\hline \multirow[b]{2}{*}{ Capitais / DF } & \multicolumn{2}{|c|}{$\begin{array}{l}\text { Atividade } \\
\text { física }\end{array}$} & \multicolumn{2}{|c|}{$\begin{array}{c}\text { Consumo abusivo } \\
\text { de álcool }\end{array}$} & \multicolumn{2}{|c|}{$\begin{array}{l}\text { IMC acima } \\
\text { de } 25 \mathrm{~kg} / \mathrm{m}^{2}\end{array}$} & \multicolumn{2}{|c|}{$\begin{array}{c}\text { Consumo de } \\
\text { frutas e hortaliças }\end{array}$} \\
\hline & $\%$ & IC $95 \%$ & $\%$ & IC 95\% & $\%$ & IC $95 \%$ & $\%$ & IC $95 \%$ \\
\hline Aracaju & 14,4 & $11,9-16,9$ & 19,5 & $16,1-22,9$ & 41,9 & $38,1-45,8$ & 18,9 & $15,9-21,8$ \\
\hline Belém & 19,8 & $16,8-22,8$ & 23,7 & $20,5-26,9$ & 45,9 & $42,3-49,5$ & 11,1 & $9,4-12,7$ \\
\hline Belo Horizonte & 16,1 & $13,7-18,4$ & 20,8 & $18,2-23,5$ & 43,3 & $39,5-47,2$ & 21,6 & $19,0-24,2$ \\
\hline Boa Vista & 20,5 & $17,1-23,9$ & 17,8 & $14,5-21,2$ & 45,7 & $41,4-50,0$ & 14,3 & $11,7-16,9$ \\
\hline Campo Grande & 18,1 & $15,4-20,7$ & 17,2 & $14,3-20,0$ & 47,5 & $44,0-51,1$ & 17,8 & $15,5-20,1$ \\
\hline Cuiabá & 15,7 & $13,1-18,2$ & 20,4 & $17,3-23,6$ & 48,4 & $44,9-51,9$ & 17,1 & $14,7-19,4$ \\
\hline Curitiba & 14,4 & $12,2-16,6$ & 10,8 & $8,8-12,8$ & 46,6 & $43,5-49,7$ & 20,7 & $18,6-22,9$ \\
\hline Florianópolis & 18,8 & $16,4-21,2$ & 17,0 & $14,7-19,3$ & 42,0 & $38,9-45,1$ & 24,1 & $21,5-26,7$ \\
\hline Fortaleza & 15,1 & $12,8-17,3$ & 17,8 & $14,6-21,0$ & 45,3 & $41,3-49,2$ & 14,8 & $12,2-17,4$ \\
\hline Goiânia & 15,7 & $13,5-18,0$ & 17,0 & $14,4-19,7$ & 44,4 & $40,4-48,5$ & 20,5 & $18,0-23,0$ \\
\hline João Pessoa & 14,7 & $12,3-17,2$ & 19,0 & $14,4-23,6$ & 45,0 & $40,4-49,5$ & 20,6 & $17,0-24,2$ \\
\hline Macapá & 19,7 & $16,3-23,0$ & 20,4 & $16,9-24,0$ & 47,7 & $43,5-52,0$ & 12,9 & $10,5-15,4$ \\
\hline Maceió & 15,6 & $12,6-18,6$ & 16,2 & $13,2-19,1$ & 42,5 & $38,2-46,8$ & 14,5 & $12,1-17,0$ \\
\hline Manaus & 15,3 & $12,8-17,8$ & 18,8 & $16,0-21,6$ & 42,1 & $38,7-45,5$ & 15,5 & $12,9-18,2$ \\
\hline Natal & 18,8 & $15,9-21,6$ & 17,5 & $14,8-20,3$ & 42,9 & $39,4-46,5$ & 19,7 & $16,9-22,5$ \\
\hline Palmas & 20,6 & $16,4-24,7$ & 23,7 & $18,9-28,5$ & 38,9 & $34,1-43,7$ & 17,9 & $14,4-21,4$ \\
\hline Porto Alegre & 15,3 & $12,5-18,1$ & 15,4 & $12,4-18,5$ & 49,0 & $45,7-52,2$ & 23,1 & $20,6-25,7$ \\
\hline Porto Velho & 15,7 & $13,3-18,1$ & 18,5 & $15,6-21,3$ & 44,1 & $40,5-47,7$ & 14,5 & $12,2-16,9$ \\
\hline Recife & 17,0 & $14,5-19,6$ & 19,2 & $16,5-22,0$ & 46,6 & $43,0-50,3$ & 20,3 & $17,5-23,0$ \\
\hline Rio Branco & 14,6 & $11,2-18,1$ & 16,5 & $12,5-20,5$ & 48,6 & $43,0-54,2$ & 12,2 & $8,7-15,7$ \\
\hline Rio de Janeiro & 15,6 & $13,4-17,9$ & 20,5 & $17,6-23,4$ & 43,8 & $40,6-46,9$ & 20,0 & $17,5-22,5$ \\
\hline Salvador & 14,5 & $12,1-16,8$ & 24,9 & $21,9-27,9$ & 41,0 & $37,8-44,2$ & 15,6 & $13,4-17,8$ \\
\hline São Luís & 15,2 & $12,8-17,6$ & 19,8 & $17,1-22,6$ & 38,7 & $35,4-41,9$ & 15,5 & $13,2-17,8$ \\
\hline São Paulo & 11,5 & $9,7-13,2$ & 12,2 & $10,2-14,3$ & 45,6 & $42,2-49,0$ & 21,8 & $19,5-24,1$ \\
\hline Teresina & 15,4 & $12,9-18,0$ & 21,6 & $18,4-24,8$ & 36,8 & $33,0-40,6$ & 13,4 & $11,2-15,5$ \\
\hline Vitória & 19,7 & $16,7-22,7$ & 18,5 & $15,5-21,4$ & 42,3 & $39,1-45,5$ & 21,3 & $18,7-23,8$ \\
\hline Distrito Federal & 19,0 & $16,3-21,7$ & 18,4 & $15,3-21,5$ & 39,6 & $36,4-42,9$ & 21,3 & $18,9-23,7$ \\
\hline
\end{tabular}

VIGITEL - Vigilância de Fatores de Risco e Proteção para Doenças Crônicas por Inquérito Telefônico; IC95\%: Intervalo de Confiança de $95 \%$. a Adultos que praticam atividades de intensidade leve ou moderada por pelo menos 30 minutos diários em 5 ou mais dias da semana ou atividades de intensidade vigorosa por pelo menos 20 minutos diários em 3 ou mais dias da semana; ${ }^{\mathrm{b}} \mathrm{Nos}$ últimos 30 dias, consumiam quatro ou mais doses (mulher) ou cinco ou mais doses (homem) de bebida alcoólica em uma mesma ocasião; 'Índice de Massa Corporal (IMC): Peso corporal (Kg)/ estatura (m) ${ }^{2}$; ${ }^{\mathrm{d}}$ Consumo de cinco ou mais porções diárias de frutas e hortaliças; ${ }^{\mathrm{e}}$ Percentual ponderado para ajustar a distribuição sócio-demográfica da amostra VIGITEL à distribuição da população adulta da cidade no Censo Demográfico de 2000. 
Tabela 4. Coeficiente de regressão linear simples $(\beta)$ e valor de $p$ associado entre prevalência de Hipertensão Arterial Sistêmica autorreferida estimada pelo VIGITELa e a padronizadab e fatores de estilo de vida e estado nutricional, VIGITEL, 2008.

\begin{tabular}{|c|c|c|c|c|}
\hline \multirow{2}{*}{ Características } & \multicolumn{2}{|c|}{$\begin{array}{c}\text { Prevalência } \\
\text { estimada } \\
\text { pelo VIGITEL }\end{array}$} & \multicolumn{2}{|c|}{$\begin{array}{c}\text { Prevalência } \\
\text { padronizada }\end{array}$} \\
\hline & $\beta$ & $\begin{array}{l}\text { valor } \\
\text { de p }\end{array}$ & $\beta$ & $\begin{array}{l}\text { valor } \\
\text { de } p\end{array}$ \\
\hline Proporção de fumantes & 0,27 & 0,22 & 0,15 & 0,28 \\
\hline Proporção de fumantes de mais de 20 cigarros por dia & 1,11 & $<0,01$ & 0,48 & 0,07 \\
\hline Proporção de indivíduos com IMCc acima de $25 \mathrm{~kg} / \mathrm{m}^{2}$ & 0,38 & 0,08 & 0,24 & 0,07 \\
\hline Proporção de obesidade & 0,65 & 0,14 & 0,44 & 0,11 \\
\hline Proporção de indivíduos com consumo recomendado de frutas e hortaliças & 0,53 & $<0,01$ & 0,19 & 0,11 \\
\hline Proporção de indivíduos que consomem o excesso de gordura da carne & $-0,09$ & 0,43 & 0,03 & 0,72 \\
\hline Proporção de indivíduos com consumo frequente de refrigerante não diet & $-0,08$ & 0,44 & $-0,03$ & 0,69 \\
\hline Proporção de indivíduos com prática regular de atividade física & $-0,55$ & 0,06 & $-0,25$ & 0,19 \\
\hline Proporção de indivíduos que relataram consumo abusivo de álcool & $-0,32$ & 0,16 & $-0,10$ & 0,47 \\
\hline Proporção de indivíduos que considera seu estado de saúde ruim & $-0,42$ & 0,45 & 0,09 & 0,79 \\
\hline Proporção de indivíduos que referiram possuir plano de saúde & 0,19 & $<0,01$ & 0,04 & 0,41 \\
\hline
\end{tabular}

VIGITEL - sistema de vigilância de fatores de risco e proteção para doenças crônicas por inquérito telefônico; ${ }^{\text {VValores }}$ ponderados para ajustar a distribuição sociodemográfica da amostra VIGITEL à distribuição da população adulta de cada cidade no Censo Demográfico de 2000; ${ }^{\text {b }}$ Padronizada pelo método direto por sexo e faixa etária da soma das 26 capitais brasileiras e Distrito Federal; cÍndice de Massa Corporal (IMC): Peso corporal (Kg)/ estatura (m) ${ }^{2}$; dIMC acima de $30 \mathrm{~kg} / \mathrm{m}^{2}$.

Tabela 5. Coeficiente de regressão linear múltipla $(\beta)$ e valor de p associado entre prevalência de Hipertensão Arterial Sistêmica autorreferida estimada pelo VIGITEL ${ }^{\mathrm{a}}$ e a padronizada ${ }^{\mathrm{b}}$ e fatores associados, mutuamente ajustados

\begin{tabular}{|c|c|c|c|c|}
\hline & \multicolumn{2}{|c|}{$\begin{array}{c}\text { Prevalência } \\
\text { estimada } \\
\text { pelo VIGITEL }\end{array}$} & \multicolumn{2}{|c|}{$\begin{array}{c}\text { Prevalência } \\
\text { padronizada }^{\mathrm{b}}\end{array}$} \\
\hline & $\beta$ & $\begin{array}{l}\text { valor } \\
\text { de p }\end{array}$ & $\beta$ & $\begin{array}{l}\text { valor } \\
\text { de } p\end{array}$ \\
\hline Proporção de indivíduos com $\mathrm{IMC}^{\mathrm{c}}$ acima de $25 \mathrm{~kg} / \mathrm{m}^{2}$ & 0,46 & 0,02 & 0,31 & 0,03 \\
\hline Proporção de indivíduos com consumo recomendado de frutas e hortaliças & 0,60 & $<0,01$ & 0,25 & 0,05 \\
\hline Proporção de indivíduos com prática regular de atividade física & $-0,55$ & 0,03 & $-0,28$ & 0,14 \\
\hline Proporção de indivíduos que relataram consumo abusivo de álcool & 0,30 & 0,18 & 0,22 & 0,20 \\
\hline Coeficiente de determinação & 0,73 & & 0,57 & \\
\hline
\end{tabular}

VIGITEL - sistema de vigilância de fatores de risco e proteção para doenças crônicas por inquérito telefônico; ${ }^{\mathrm{a}}$ Valores ponderados para ajustar a distribuição sociodemográfica da amostra VIGITEL à distribuição da população adulta de cada cidade no Censo Demográfico de 2000; ${ }^{b}$ Padronizada pelo método direto por sexo e faixa etária da soma das 26 capitais brasileiras e Distrito Federal. ' 'Índice de Massa Corporal (IMC): Peso corporal (Kg)/ estatura $(\mathrm{m})^{2}$.

derada, pode ter ocorrido pelo efeito de diferentes distribuições de idade nas populações das cidades estudadas.

A prevalência de HAS autorreferida foi maior no sexo feminino e faixas etárias mais elevadas. Este resultado pode ser devido ao fato de que mulheres utilizam os serviços de saúde com maior frequência e, consequentemente, há maior pro- porção de diagnósticos médicos neste sexo ${ }^{10}$. Além disso, existem evidências de que a idade e a prevalência de HAS estão diretamente relacionadas ${ }^{15}$. Segundo Vasan et al. ${ }^{16}$, pessoas com idade entre 55 e 65 anos têm 90\% de chance de desenvolver HAS ao longo da vida. Resultados do $\mathrm{Na}$ tional Health and Nutrition Examination Survey (NHANES) verificaram aumento da HAS com a 
idade, variando de $7 \%$ entre aqueles com idade entre 18-39 anos para 67\% entre aqueles com 60 anos ou mais ${ }^{17}$.

Ferreira et al. ${ }^{18}$ ao avaliarem dados do VIGITEL, coletados em 2006, observaram resultados semelhantes aos encontrados no presente estudo, demonstrando o seguimento das características associadas à prevalência de HAS. Estes autores verificaram que a frequência de HAS autorreferida foi maior entre mulheres $(24,4 \% v s$ $18,4 \%)$ e nas faixas etárias mais elevadas, além disso, a chance de ter hipertensão, ajustada para variáveis de confusão, foi maior entre os indivíduos com excesso de peso e outras morbidades. Os autores também encontraram associação estatisticamente significante entre a presença de HAS e a prática de caminhada em ambos os sexos.

Tendo em vista as diferenças na distribuição etária e quanto ao sexo entre as capitais brasileiras e Distrito Federal e que o risco de desenvolver HAS aumenta consideravelmente com a idade, a padronização da prevalência de HAS autorreferida pelo sexo e faixa etária permitiu realizar comparações entre as cidades estudadas retirando, ou atenuando, o efeito desses fatores sobre essa estimativa.

A associação positiva entre proporção de excesso de peso e prevalência de HAS nas 26 capitais brasileiras e Distrito Federal evidencia a importante relação entre o estado nutricional e essa morbidade, mesmo em nível ecológico. Estimase que entre 20 a $30 \%$ da prevalência de HAS pode ser explicada pela associação existente entre excesso de peso e aumento do risco de $\mathrm{HAS}^{19,20}$. Uma relação de causa e efeito entre aumento da massa corporal e elevação da pressão arterial já foi demonstrada em vários estudos de coorte ${ }^{21-}$ ${ }^{23}$. Na coorte dos descendentes de Framingham foi observado aumento da incidência de HAS diretamente proporcional ao aumento da massa corporal $^{24}$.

Em nível individual, a dieta tem papel importante entre os fatores relacionados ao estilo de vida que corroboram para a elevação dos níveis da pressão arterial. A ingestão elevada de sódio e álcool e o baixo consumo de potássio e fibras estão relacionados com o aumento dos níveis pressóricos $^{25}$, assim como o consumo excessivo de açúcar que, além de suas calorias vazias, tendo a obesidade como marcador, apresenta também efeito "tóxico" sobre o organismo, sendo responsável pelo desenvolvimento de outras doenças crônicas, tais como as doenças cardíacas ${ }^{26}$.

Evidências associam o consumo insuficiente de frutas e hortaliças ao risco elevado para ocor- rência de doenças, como certos tipos de câncer e doenças cardiovasculares ${ }^{27,28}$. Entretanto, neste estudo, houve associação positiva entre a proporção de indivíduos que referiram consumo recomendado de frutas e hortaliças e a prevalência de HAS nas 27 cidades estudadas. Como a população, de um modo geral, reconhece que uma alimentação saudável inclui maior ingestão de frutas e hortaliças, sendo uma medida eficaz para a prevenção e controle tanto do excesso de peso como de doenças crônicas como HAS, há a possibilidade do efeito da causalidade reversa para essa associação positiva, podendo ser devido a uma forma de controle de morbidades.

Apesar de não observada associação entre a proporção de indivíduos que referiram consumo abusivo de álcool e a prevalência de HAS, o consumo de bebidas alcoólicas é um fator comportamental de risco ${ }^{21,29}$. De acordo com o estudo de Martinez e Latorre ${ }^{30}$, indivíduos que faziam uso de bebida alcoólica diariamente apresentaram 3,0 vezes mais chance de estarem hipertensos quando comparados aos não etilistas.

Quanto a variáveis socioeconômicas, em nosso estudo utilizamos a proporção de indivíduos que possuíam plano de saúde como um fator socioeconômico, porém essa variável mostrou associação positiva apenas com a proporção de HAS ponderada na análise univariada. Quando incluído nos modelos múltiplos, este fator não apresentou associação estatisticamente significantemente de desfecho e não alterou o coeficiente de associação das demais variáveis, assim como o coeficiente de determinação do modelo (R2). Entretanto, Ishitani et al. ${ }^{31}$ por meio de um estudo ecológico que analisou mortalidade entre 1999 a 2001, por doenças cardiovasculares e pelos subgrupos das doenças isquêmicas do coração e cerebrovasculares-hipertensivas entre adultos de 98 municípios de todas as macrorregiões brasileiras, obtidos pelo Sistema de informação sobre Mortalidade, observaram associação inversa entre condições socioeconômicas (indicadas pela renda, escolaridade e moradia) e mortalidade por essas doenças.

As associações verificadas neste estudo, não são de resultado imediato, como a observada entre HAS e proporção de indivíduos com excesso de peso, por exemplo. Provavelmente elas estão ocorrendo há algum tempo, porém como este estudo trabalha com um corte transversal, os possíveis fatores de risco ou proteção são vistos em um mesmo momento, impossibilitando a utilização do tempo como critério para avaliação dos efeitos da prevalência desses fatores. 
Apesar disso, resultados semelhantes foram encontrados por Borges et al. ${ }^{32}$ ao avaliarem amostra probabilística de adultos de Belém (PA), utilizando dados do SIMTEL (monitoramento de doenças crônicas por telefone) em 2005, e observaram que a prevalência de HAS se associou diretamente com a idade e com o excesso de peso, sendo maior entre as mulheres, assim como no presente estudo.

$\mathrm{Na}$ interpretação dos resultados deste trabalho, deve-se considerar que se trata de um estudo ecológico, onde a pergunta que se deseja responder, não é sobre as causas dos casos de hipertensos, mas sobre as causas da prevalência de HAS em grupos populacionais, comparando, neste caso, as populações das 26 capitais brasileiras e do Distrito Federal. Cabe ressaltar que, segundo a estimativa do IBGE de 2008, disponível no DATASUS $^{13}, 24,6 \%$ de todos os adultos do país vivem nessas capitais. Entretanto, a comparação com estudos ecológicos é prejudicada pela escassez desses tipos de estudos na literatura nesta área.

Além disso, entre as limitações deste estudo, pode-se citar a obtenção da informação sobre HAS autorreferida e a representatividade da amostra estudada, restrita a indivíduos que possuíam telefone fixo. Entretanto, a HAS autorreferida tem se mostrado um indicador adequado para as estimativas de sua frequência nas populações, com as vantagens de rapidez e baixo custo na obtenção da informação $0^{10,33}$.

Quanto à representatividade da amostra, é importante considerar que a de adultos entrevistada pelo VIGITEL em cada cidade é extraída a partir do cadastro das linhas telefônicas residenciais existentes e, portanto, só permite inferências populacionais para a população adulta que reside em domicílios cobertos pela rede de telefonia fixa. Embora a cobertura desta rede tenha crescido nos últimos anos, não é evidentemente universal. A partir da Pesquisa de Orçamentos Familiares (POF) realizada pelo IBGE entre 2002 e 2003, estimou-se que 66,4\% dos domicílios existentes no conjunto das 26 capitais brasileiras e Distrito Federal eram servidos por linhas telefônicas fixas, variando entre 33,7\% em Macapá e $79,4 \%$ no Distrito Federal ${ }^{12}$.

Apesar de não ter sido empregado um método direto para a compensação dos domicílios não servidos por linhas telefônicas, este viés foi minimizado por meio da utilização dos fatores de expansão para representar a população adulta total das cidades estudadas, segundo a distribuição sociodemográfica do censo de 2000, incluindo sexo, idade e nível de escolaridade ${ }^{12}$. Ain- da assim, essa correção apenas "aproxima” as estimativas geradas pelo sistema das que seriam observadas caso a cobertura da rede telefônica fosse universal. A aproximação será tanto maior quanto mais decisiva for a influência do sexo, idade e nível de escolaridade sobre a frequência de interesse, como é o caso da Hipertensão Arterial autorreferida.

Em relação aos custos em inquéritos epidemiológicos, Monteiro et al. ${ }^{34}$ apontam que o custo por entrevista, no sistema de monitoramento de fatores de risco para DCNT no município de São Paulo, feita em 2005, foi em média de $\mathrm{R} \$$ 10,90 por entrevista realizada, sendo oito vezes inferior ao custo estimado em sistemas existentes em países desenvolvidos, tal como o sistema americano BRFSS (Behavioral Risk Factor Surveillance System), estimado em 25 a 30 dólares (ou cerca de $\mathrm{R} \$ 90,00$ ) e quatro a oito vezes inferior ao custo de inquéritos domiciliares tradicionais realizados no Município de São Paulo, cujo custo médio por entrevista foi entre R\$50,00 e $\mathrm{R} \$ 100,00$.

Portanto, considerando o alto custo e a difícil operacionalização de inquéritos domiciliares regulares com medidas objetivas da pressão arterial, as informações resultantes do VIGITEL podem contribuir para o planejamento, monitoramento e avaliação das ações nacionais para seu controle.

\section{Conclusões}

A prevalência de HAS observada neste estudo variou entre as 27 cidades estudadas, sendo menor em Palmas e maior no Rio de Janeiro. Esta prevalência foi mais elevada entre o sexo feminino e faixas etárias mais avançadas em todas as capitais e Distrito Federal.

Neste estudo verificamos associação positiva entre a prevalência de HAS, tanto ponderada quanto padronizada, e a proporção de indivíduos com excesso de peso, assim como com a proporção que referiram consumo recomendado de frutas e hortaliças. Esta última associação pode ser devido a causalidade reversa, visto que é de conhecimento da população que o consumo desses grupos alimentares é uma medida eficaz para a prevenção e o controle do excesso de peso e de doenças crônicas como HAS.

A associação positiva com a proporção de indivíduos com excesso de peso alerta para a prevenção e o controle deste importante fator de risco para diversas morbidades, como HAS. Vis- 
to que nos últimos anos o país vem passando pela transição nutricional, com o aumento da prevalência de sobrepeso e obesidade e diminuição da atividade física, não só na população adulta como também entre crianças e adolescentes, estratégias de saúde pública devem ser instituídas para a prevenção, controle e tratamento da HAS e do excesso de peso não só na população adulta, como também em populações mais jovens, a fim de reduzir os prejuízos à saúde a curto e a longo prazo.

\section{Colaboradores}

AP Muraro, PRM Rodrigues e DF dos Santos participaram de todas as etapas da pesquisa: concepção, delineamento, busca e análise dos dados, interpretação dos resultados e redação do artigo. JU Braga participou da concepção, desenho, análise e interpretação dos dados e revisão crítica do artigo. Todos os autores aprovaram a versão final a ser publicada.

\section{Agradecimentos}

Aos professores Gulnar Azevedo, Washington Leite Junges e Joaquim Valente pelas sugestões para este estudo. 


\section{Referências}

1. Lewington S, Clarke R, Qizilbash N, Peto R, Collins R. Age-specific relevance of usual blood pressure to vascular mortality: a meta-analysis of individual data for one million adults in 61 prospective studies. Lancet 2002; 360(9349):1903-1913.

2. Kearney PM, Whelton M, Reynolds K, Muntner P, Whelton PK, He J. Global burden of hypertension: analysis of worldwide data. Lancet 2005; 365(9455): 217-223.

3. Instituto Brasileiro de Geografia e Estatística (IBGE). Pesquisa nacional por amostra de domicílios (PNAD 2008), um panorama da Saúde no Brasil: acesso e utilização dos serviços, condições de saúde e fatores de risco e proteção à saúde. Rio de Janeiro: IBGE; 2010.

4. Brasil. VIGITEL Brasil 2009: vigilância de fatores de risco e proteção para doenças crônicas por inquérito telefônico. In: Secretaria de Vigilância em Saúde. Secretaria de Gestão Estratégica e Participativa. Brasília: Ministério da Saúde (MS); 2010.

5. Schmidt MI, Duncan BB, Azevedo e Silva G, Menezes AM, Monteiro CA, Barreto SM, Chor D, Menezes PR. Chronic non-communicable diseases in Brazil: burden and current challenges. Lancet 2011; 377(9781):1949-1961.

6. Sociedade Brasileira de Cardiologia (SBC), Sociedade Brasileira de Hipertensão (SBH), Sociedade Brasileira de Nefrologia (SBN). VI Diretrizes Brasileiras de Hipertensão. Arq Bras Cardiol 2010; 95(1):1-51.

7. Coitinho DCL, Mendonça M, Recine E, Sichieri R. Condições nutricionais da população brasileira: adultos e idosos: pesquisa nacional sobre saúde e nutrição. Brasília: Instituto Nacional de Alimentação e Nutrição (INAN); 1991

8. Jardim PC, Gondim Mdo R, Monego ET, Moreira HG, Vitorino PV, Souza WK, Scala LC. High blood pressure and some risk factors in a Brazilian capital. Arq Bras Cardiol 2007; 88(4):452-457.

9. Chrestan MAD, Santos IS, Matijasevich AM. Hipertensão Arterial Sistêmica autorreferida: validação diagnóstica em estudo de base populacional. Cad Saude Publica 2009; 25(11):2395-2406.

10. Lima-Costa MF, Peixoto SV, Firmo JO. Validade da hipertensão arterial autorreferida e seus determinantes (projeto Bambuí). Rev Saude Publica 2004; 38(5) 637-642.

11. Moura EC, Neto OLM, Malta DC, Moura L, Nunes da Silva N, Bernal R, Claro RM, Monteiro CA. Vigilância de Fatores de Risco para Doenças Crônicas por Inquérito Telefônico nas capitais dos 26 estados brasileiros e no Distrito Federal (2006). Rev Bras Epidemiol 2008; 11(Supl. 1):20-37.

12. Brasil. VIGITEL Brasil 2008: vigilância de fatores de risco e proteção para doenças crônicas por inquérito telefônico. In: Secretaria de Vigilância em Saúde. Secretaria de Gestão Estratégica e Participativa. Brasília: Ministério da Saúde (MS); 2009.

13. Brasil. Ministério da Saúde (MS). Departamento de Informática do SUS. DATASUS [acessado 2011 ago 15]. Disponível em: http://www.datasus.gov.br

14. The R Foundation for Statistical Computing. [página na Internet]. [acessado 2013 mar 26]. Disponível em: http://www.R-project.org
15. Dórea EL, Lotufo PA. Framingham Heart Study e a teoria do contínuo de Pickering: duas contribuições da epidemiologia para a associação entre pressão arterial e doença cardiovascular. Rev Bras Hipertens 2001; 8(2):195-199.

16. Vasan RS, Larson MG, Leip EP, Kannel WB, Levy D. Assessment of frequency of progression to hypertension in non-hypertensive participants in the Framingham Heart Study: a cohort study. Lancet 2001; 358(9294):1682-1686.

17. Ostchega Y, Yoon SS, Hughes J, Louis T. Hypertension awareness, treatment, and control-continued disparities in adults: United States, 2005-2006. NCHS Data Brief 2008(3):1-8.

18. Ferreira SRG, Moura EC, Malta DC, Sarno F. Frequência de hipertensão arterial e fatores associados: Brasil, 2006. Rev Saude Publica 2009; 43(2):98-106.

19. Fung TT, Rimm EB, Spiegelman D, Rifai N, Tofler GH, Willett WC, Hu FB. Association between dietary patterns and plasma biomarkers of obesity and cardiovascular disease risk. Am J Clin Nutr 2001; 73(1):61-67.

20. Millen BE, Quatromoni PA, Copenhafer DL, Demissie S, O'Horo CE, D'Agostino RB. Validation of a dietary pattern approach for evaluating nutritional risk: the Framingham Nutrition Studies. $J$ Am Diet Assoc 2001; 101(2):187-194.

21. Brummett BH, Babyak MA, Siegler IC, Shanahan M, Harris KM, Elder GH, Williams RB. Systolic blood pressure, socioeconomic status, and biobehavioral risk factors in a nationally representative US young adult sample. Hypertension 2011; 58(2):161-166.

22. Ix JH, Biggs ML, Kizer JR, Mukamal KJ, Djousse L, Zieman SJ, de Boer IH, Nelson TL, Newman AB, Criqui MH, Siscovick DS. Association of body mass index with peripheral arterial disease in older adults: the Cardiovascular Health Study. Am J Epidemiol 2011; 174(9):1036-1043.

23. Juhola J, Magnussen CG, Viikari JS, Kahonen M, Hutri-Kahonen N, Jula A, Lehtimaki T, Akerblom HK, Pietikainen M, Laitinen T, Jokinen E, Taittonen L, Raitakari OT, Juonala M. Tracking of serum lipid levels, blood pressure, and body mass index from childhood to adulthood: the Cardiovascular Risk in Young Finns Study. J Pediatr 2011; 159(4):584-590.

24. Lamon-Fava S, Wilson PW, Schaefer EJ. Impact of body mass index on coronary heart disease risk factors in men and women. The Framingham Offspring Study. Arterioscler Thromb Vasc Biol 1996; 16(12):1509-1515.

25. Velásquez-Meléndez G, Barreto SM, Pimenta AM. Fatores nutricionais e hipertensão arterial. Epidemiologia Nutricional. Rio de Janeiro: Fiocruz, Atheneu; 2007.

26. Lustig RH, Schmidt LA, Brindis CD. Public health: The toxic truth about sugar. Nature 2012; 482(7383): 27-29.

27. Liu S, Manson JE, Lee IM, Cole SR, Hennekens $\mathrm{CH}$, Willett WC, Buring JE. Fruit and vegetable intake and risk of cardiovascular disease: the Women's Health Study. Am J Clin Nutr 2000; 72(4):922-928. 
28. Van Duyn MA, Pivonka E. Overview of the health benefits of fruit and vegetable consumption for the dietetics professional: selected literature. J Am Diet Assoc 2000; 100(12):1511-1521.

29. Mancia G, De Backer G, Dominiczak A, Cifkova R, Fagard R, Germano G, Grassi G, Heagerty AM, Kjeldsen SE, Laurent S, Narkiewicz K, Ruilope L, Rynkiewicz A, Schmieder RE, Boudier HA, Zanchetti A. 2007 ESH-ESC Practice Guidelines for the Management of Arterial Hypertension: ESH-ESC Task Force on the Management of Arterial Hypertension. J Hypertens 2007; 25(9):1751-1762.

30. Martinez MC, Latorre MRDO. Fatores de risco para hipertensão arterial e diabete melito em trabalhadores de empresa metalúrgica e siderúrgica. Arq Bras Cardiol 2006; 87(4):471-479.

31. Ishitani LH, Franco GC, Perpétuo IHO, Franca E. Desigualdade social e mortalidade precoce por doenças cardiovasculares no Brasil. Rev Saude Publica 2006; 40(4):684-691.

32. Borges HP, Cruz Ndo C, Moura EC. Association between hypertension and overweight in adults in Belem, state of Para (Brazil), 2005. Arq Bras Cardiol 2008; 91(2):99-106.

33. Ferreira AD, Cesar CC, Malta DC, Andrade AC, Ramos CG, Proietti FA, Bernal RT, Caiaffa WT. Validity of data collected by telephone survey: a comparison of VIGITEL 2008 and 'Saude em Beaga' survey. Rev Bras Epidemiol 2011; 14(Supl. 1):16-30.

34. Monteiro CA, de Moura EC, Jaime PC, Lucca A, Florindo AA, Figueiredo IC, Bernal R, da Silva NN. Monitoramento de fatores de risco para doenças crônicas por entrevistas telefonicas. Rev Saude Publica $2005 ; 39(1): 47-57$.

Artigo apresentado em 03/02/2012

Aprovado em 02/04/2012

Versão final apresentada em 25/04/2012 